\title{
Zero-Field NMR Enhanced by Parahydrogen in Reversible Exchange
}

Thomas Theis, ${ }^{*}, \dagger$ Micah P. Ledbetter, ${ }^{\S}$ Gwendal Kervern, ${ }^{\dagger, \ddagger}$ John W. Blanchard, ${ }^{\dagger, \ddagger}$ Paul J. Ganssle, ${ }^{\dagger, \ddagger}$ Mark C. Butler, ${ }^{\dagger, \ddagger}$ Hyun D. Shin, ${ }^{\ddagger}$ Dmitry Budker, ${ }^{\S}, \|$ and Alexander Pines $*, \dagger, \ddagger$

\author{
${ }^{\dagger}$ Materials Science Division and "Nuclear Science Division, Lawrence Berkeley National Laboratory, Berkeley California 94720 , \\ United States \\ ${ }^{\ddagger}$ Department of Chemistry, University of California at Berkeley, Berkeley, California 94720-3220, United States \\ ${ }^{\S}$ Department of Physics, University of California at Berkeley, Berkeley, California 94720-7300, United States
}

\section{Supporting Information}

ABSTRACT: We have recently demonstrated that sensitive and chemically specific NMR spectra can be recorded in the absence of a magnetic field using hydrogenative parahydrogen induced polarization (PHIP $)^{1-3}$ and detection with an optical atomic magnetometer. Here, we show that non-hydrogenative parahydrogen-induced polarization ${ }^{4-6}$ (NH-PHIP) can also dramatically enhance the sensitivity of zero-field NMR. We demonstrate the detection of pyridine, at concentrations as low as $6 \mathrm{mM}$ in a sample volume of $250 \mu \mathrm{L}$, with sufficient sensitivity to resolve all identifying spectral features, as supported by numerical simulations. Because the $\mathrm{NH}$ PHIP mechanism is nonreactive, operates in situ, and eliminates the need for a prepolarizing magnet, its combination with optical atomic magnetometry will greatly broaden the analytical capabilities of zero-field and low-field NMR.

$\mathrm{T}$ he past decade has witnessed increasing interest in the development of low-cost portable NMR spectrometers. ${ }^{7}$ Such spectrometers promise to enable chemical analysis at greatly reduced cost in environments not accessible to standard high-field NMR technology. Detection of analytes at low concentrations primarily requires sensitive detectors and sufficient nuclear polarization. The weak thermal polarization of nuclear spins has given NMR the reputation of being an inherently insensitive method. For example, even in magnetic fields of superconducting magnets, the polarization obtained does not exceed $10^{-4}$.

A variety of available hyperpolarization techniques such as dynamic nuclear polarization (DNP), ${ }^{8,9}$ chemically induced DNP (CIDNP), ${ }^{10}$ spin-exchange optical pumping $(\mathrm{SEOP})^{11-13}$ of noble gases, and parahydrogen induced polarization (PHIP) $)^{1-3,14,15}$ suggest that sensitivity limitations given by the Boltzmann thermal polarization can be overcome for a large range of analytes. All these hyperpolarization techniques, DNP, ${ }^{16-19} \mathrm{CIDNP}^{20-22} \mathrm{SEOP}^{23}$ and PHIP, ${ }^{24,25}$ have been shown to greatly enhance sensitivity of low-field NMR experiments where thermal polarization is even lower.

Of equal importance, sensitive low-field NMR detectors of nuclear magnetization have been developed. These include systems based on inductive detectors, ${ }^{7,26,27}$ superconducting quantum-interference devices (SQUIDs),,$^{19,28-30}$ and optical magnetometers. ${ }^{24,31-34}$ These technologies, in varying stages of maturity, permit the sensitive detection of low-frequency NMR signals, including NMR spectra in Earth's magnetic field.

Combinations of hyperpolarization and novel detection schemes are thus particularly attractive in unconventional or portable NMR applications. For example, we have recently demonstrated that high-resolution and high SNR spectra can be recorded at zero field using PHIP from standard hydrogenative processes and an atomic magnetometer. ${ }^{24}$ In the present contribution, we describe the first NMR experiments in zero field employing non-hydrogenative parahydrogen induced polarization (NH-PHIP) producing signal amplification by reversible exchange (SABRE). ${ }^{4-6}$ Following the approach used in refs 24,31 , and 35 , we work at zero magnetic field by eliminating the Earth's magnetic field with $\mu$-metal shields, and we use an all-optical rubidium magnetometer for detection. In addition to cryogen-free and potentially portable nature of our technique, the magnetically shielded environment has high absolute spatial and temporal homogeneity. We demonstrate that this technique enables the detection of small quantities of analyte (down to $6 \mathrm{mM}$ in a sample volume of $250 \mu \mathrm{L}$, i.e., 100 $\mathrm{nL}$ as neat liquid) while retaining all analytically useful spectral information of the zero-field spectrum. The sensitivity of the present experiments is also illustrated by the first observation of zero field NMR signals from nitrogen-15 in natural abundance. In contrast to previous work at high magnetic field ${ }^{4-6}$ and at low magnetic field, ${ }^{25,36}$ the NH-PHIP process presented here evolves entirely at zero field. The spectra exhibit significant shifts in line positions as a function of the molecular environment, in this case, due to solvent effects. The acquired spectra are in agreement with computer-simulated spectra, showing the dependence on the topology and parameters of the $J$-coupling network and demonstrating the high information content of zero-field spectra. It is important to note that the NH-PHIP method is not limited to a specific substrate but can be used on a growing class of compounds containing nitrogenheterocycles ${ }^{4,37}$ as well as amino acids and peptides. ${ }^{38}$

The experimental setup employed here was similar to that used in previous work. ${ }^{24,31}$ The sample was contained in a standard $5 \mathrm{~mm}$ NMR tube placed directly above a zero-field alkali-vapor magnetometer. This detector consists of microfabricated $5 \mathrm{~mm} \times 2 \mathrm{~mm} \times 1 \mathrm{~mm}$ rubidium cell, thereby

Received: November 30, 2011

Published: February 14, 2012 
reducing much of the size requirements associated with traditional high-field NMR. The sample, with a total volume of $250 \mu \mathrm{L}$, was composed of pyridine (Sigma CAS\# 34322-457) and Crabtree's catalyst (1,5-cyclooctadiene)(pyridine)(tricyclohexylphosphine)-iridium(I) hexafluorophosphate (Sigma CAS\# 64536-78-3) in anhydrous methanol (Sigma CAS\# 67-56-1) as solvent. The sample was held at a temperature of $40{ }^{\circ} \mathrm{C}$. The tube was connected to a gas manifold and parahydrogen was bubbled through the solution at a flow rate of $50 \mathrm{sccm}$ at a pressure of 70 psig (5.8 bar). Bubbling was stopped $0.3 \mathrm{~s}$ prior to acquisition. All measurements were performed at a catalyst-to-analyte ratio of $1: 6$ by weight (following ref 25.). The sensitivity of the magnetometer to fields in the vertical direction (i.e., the direction of detection) is about $1 \mathrm{nG} / \mathrm{Hz}^{1 / 2}$ in the frequency range of $0-20 \mathrm{~Hz}$, where signals occur in the present work.

Figure 1 illustrates the NH-PHIP transfer process, which occurs when parahydrogen and the analyte are in reversible

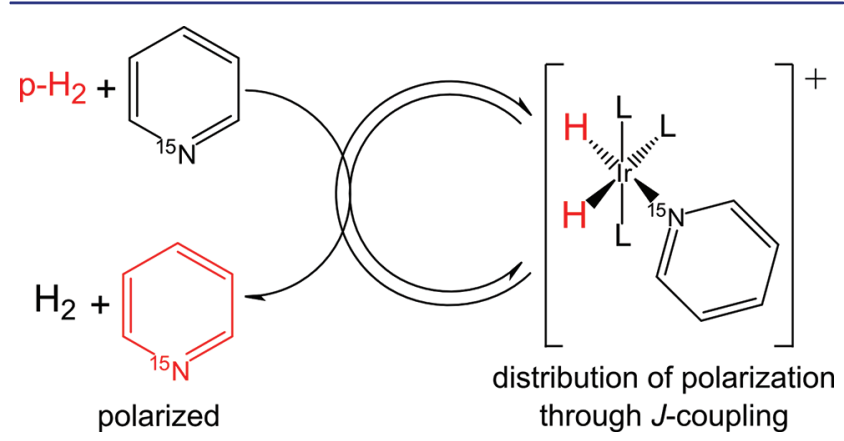

Figure 1. NH-PHIP transfer mechanism. Hydrogen and pyridine bind reversibly to the catalytic intermediate. Polarization is transferred from parahydrogen to pyridine through the network of J-couplings.

exchange via an iridium-based catalytic intermediate. During the time period in which an analyte molecule and a parahydrogen molecule are simultaneously bound to the same metal complex, a J-coupling network is formed, causing the singlet spin order of parahydrogen to spread throughout the metal complex; in particular, scalar spin order develops among the spins of the analyte, which persists in the analyte after the complex dissociates. ${ }^{5}$ This scalar spin order is a form of polarization not bearing magnetization and without directionality, since there is no magnetic field along which alignment of the polarization could occur. Fortunately, spin order involving heteronuclear pairs can be converted to observable magnetization by application of a DC magnetic-field pulse. A coil is used to apply these pulses in the vertical direction. Such a DC pulse breaks the symmetry of the initial state for heteronuclear pairs because it rotates nuclei with differing gyromagnetic ratio by differing angles. Thereby the pulse creates the coherences whose ensuing evolution produce observable oscillations of the vertical component of magnetization. The duration of the pulse is short and the amplitude is strong compared to the $J$ couplings such that evolution under the J-couplings can be ignored during the pulse. Scalar spin order transferred to homonuclear ${ }^{1} \mathrm{H}$ pairs is not excitable because it has singlet character and the symmetry is not broken by a pulse acting on nuclei with identical gyromagnetic ratio. The Supporting Information (SI) provides a more detailed description of the experimental setup, as well as a discussion of the polarization process. Additionally, a two-spin model is presented that describes the effect of the DC-pulse on a heteronuclear singlet and the ensuing evolution under the $J$-coupling Hamiltonian.

A single-shot transient and the imaginary part of its Fourier transform obtained with ${ }^{15} \mathrm{~N}$-labeled pyridine are shown by the solid blue curves in Figure 2a,b. The single-shot signal-to-noise
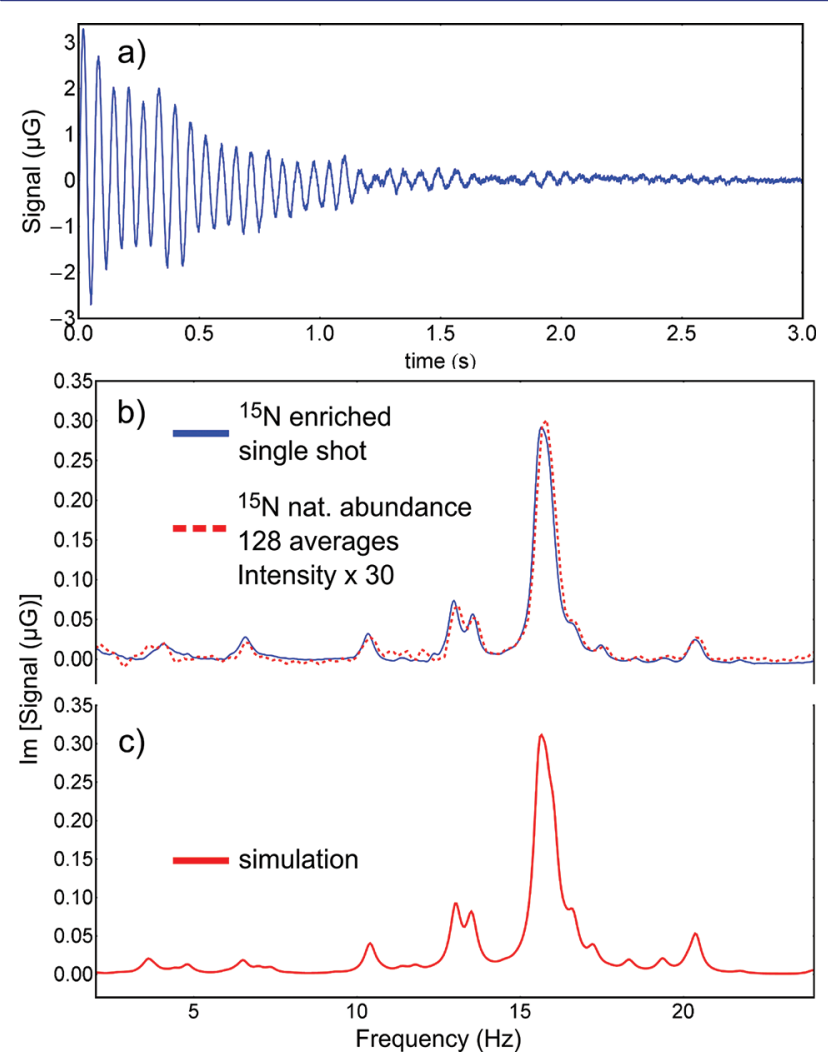

Figure 2. (a) Single-shot transient and (b) the imaginary part of its Fourier transform for NH-PHIP polarized pyridine. The solid blue trace in panels a and b shows data acquired with a $250 \mu \mathrm{L}$ sample after $10 \mathrm{~s}$ of bubbling parahydrogen through a solution containing Crabtree's catalyst $(0.6 \mathrm{mM})$, with methanol as solvent and ${ }^{15} \mathrm{~N}$ labeled pyridine as analyte at a concentration of $40 \mathrm{mM}$. (b) Spectrum (red-dashed line) obtained from pyridine at natural abundance of ${ }^{15} \mathrm{~N}$ $(0.36 \%)$ at a concentration of $3 \mathrm{M}$, which implies a $10 \mathrm{mM}$ concentration of ${ }^{15} \mathrm{~N}$-containing pyridine. (c) A numerically simulated spectrum is provided for comparison.

of the largest peak is close to 300 at a noise floor of $1 \mathrm{nG}$. The dashed red curve in Figure $2 \mathrm{~b}$ shows that it is possible to acquire identical spectra from pyridine with ${ }^{15} \mathrm{~N}$ at its low natural abundance of $0.36 \%$. For the unlabeled sample, the concentration of the analyte was larger by a factor of 75 than for the labeled sample.

Note, however, that the signal does not scale linearly with the concentration of the analyte but is dependent on many parameters such as exchange rates and the concentrations of parahydrogen, catalyst, and analyte.

The spectrum can be approximately understood as follows: The largest $J$-coupling in ${ }^{15} \mathrm{~N}$-pyridine is the two-bond coupling, ${ }^{2} J_{\mathrm{NH}}$, between the ${ }^{15} \mathrm{~N}$ and the two equivalent nearest protons, approximately forming an $\mathrm{AX}_{2}$ system. Such a system yields a peak at $3 J / 2 .{ }^{31}$ Since ${ }^{2} J_{\mathrm{NH}}=10.14 \mathrm{~Hz}$ (see Supporting Information (SI)), we expect signal at $\sim 15.3 \mathrm{~Hz}$. Long-range couplings to other protons produce a number of additional sidebands and a small shift of the largest peak to $\sim 15.6 \mathrm{~Hz}$. Note that the imaginary component of the Fourier transform is 
shown (without additional phasing). In these NH-PHIP experiments, the imaginary part is absorptive because the evolution starts from a nonmagnetized state (see SI). These features are also reproduced by a simulation shown in the solid red trace Figure 2c. (Details concerning the simulation are provided in the SI.) Interestingly, despite the excellent SNR for the ${ }^{15} \mathrm{~N}$ containing isotopomers of pyridine, it was not possible to detect ${ }^{13} \mathrm{C}$-pyridine isotopomers at their natural abundance (see SI). We attribute this to the low (1.1\%) abundance of ${ }^{13} \mathrm{C}$ and the higher signal complexity in isotopomers with both ${ }^{13} \mathrm{C}$ and ${ }^{15} \mathrm{~N}$, as discussed in the SI.

For comparison with the NH-PHIP experiments, we performed measurements of thermally polarized ${ }^{15} \mathrm{~N}$-labeled pyridine. These experiments were performed with an identical 5 mm NMR tube. A sample of $250 \mu \mathrm{L}$ neat $(14 \mathrm{M}){ }^{15} \mathrm{~N}$-labeled pyridine was polarized in a $1.6 \mathrm{~T}$ permanent magnet and then pneumatically shuttled to the zero-field region for detection. ${ }^{35}$ The time for transfer of the sample from the magnet into the detection region was approximately $250 \mathrm{~ms}$. The NMR tube traveled in a solenoid that provided a "guiding field," ensuring that the spins remained magnetized parallel to the guiding field during the shuttling. Upon arrival of the sample in the zero-field region, the guiding field was turned off suddenly with respect to any spin dynamics that occur under the influence of the $J$ couplings, and the spectrum shown in Figure 3 was acquired.

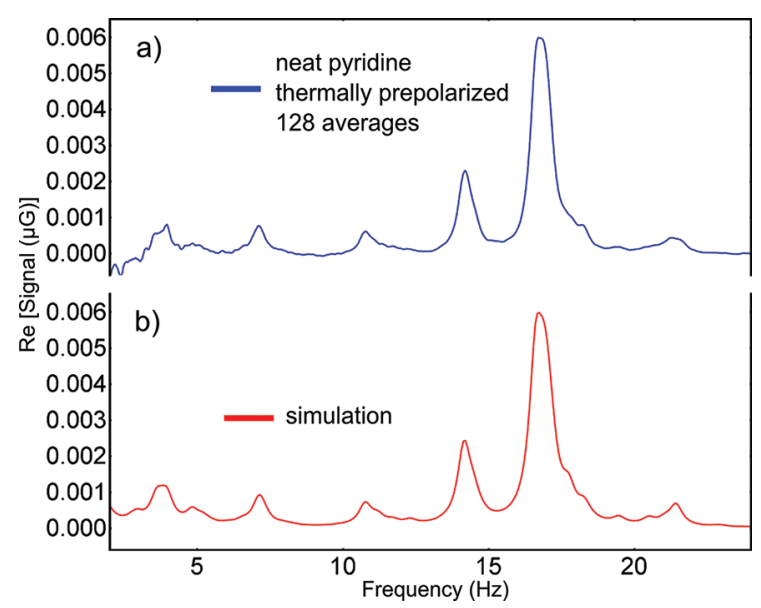

Figure 3. Spectrum obtained after averaging 128 transients of neat ${ }^{15} \mathrm{~N}$-labeled pyridine, prepolarized in a $1.6 \mathrm{~T}$ permanent magnet and shuttled into the zero-field region for detection. (a) Experimental data; (b) simulated spectrum.

Note that for this experiment, evolution starts from a magnetized state and it is the real part of the Fourier transform that is absorptive, as in conventional ID NMR. (see SI.)

After averaging 128 transients, the SNR of the main feature centered near $17 \mathrm{~Hz}$ is $\sim 70$. Comparison of this value to the single-shot SNR of $\sim 300$ for the NH-PHIP measurements gives an estimate of the achieved sensitivity enhancement: scaling by $\sqrt{ } 128$ for the number of averages and by $14 \mathrm{M} / 40 \mathrm{mM}$ for the difference in concentration reveals a sensitivity gain of $\sim 1.7 \times$ $10^{4}$ with NH-PHIP.

Additionally, a frequency shift of the main peak of (1.2 \pm $0.1) \mathrm{Hz}$ is observed. For NH-PHIP measurements in methanol, the main peak is centered around $15.6 \mathrm{~Hz}$ and shifts to $16.8 \mathrm{~Hz}$ in the neat liquid. The frequency shift is presumably due to the change in the microscopic environment associated with the presence of the solvent. This effect has been documented in detail in measurements performed at high field with cryogenically cooled magnets, ${ }^{39,40}$ but the experiments presented here demonstrate that similar information can be extracted using zero-field spectroscopy with optical detection.

Finally, we show that NH-PHIP is particularly promising for analysis of compounds in low concentration. Table 1 provides the signal-to-noise ratio obtained for five different concentrations of ${ }^{15} \mathrm{~N}$-pyridine.

All measurements were performed at a catalyst-to-analyte ratio of $1: 6$ by weight. For concentrations down to $40 \mathrm{mM}$, the signal appears independent of the amount of analyte, and is presumably dictated by the amount of dissolved parahydrogen. This is in agreement with the findings by Gong et al. ${ }^{25}$ At a concentration of $6 \mathrm{mM}$, the main peak at $\sim 15.6 \mathrm{~Hz}$ has an SNR of $\sim 15$. At that concentration, smaller peaks that are $10-30$ times weaker than the main peak have an SNR on the order of one, and spectral information is lost at lower concentrations. At the expense of spectral information, it is possible to go to even lower concentrations, as demonstrated in ref 25 .

In conclusion, we have demonstrated that NH-PHIP can be used in zero-field NMR spectroscopy. Polarization, in the form of scalar order, can be obtained at zero magnetic field, which can be converted to magnetization solely through the presence of a heteronucleus. The sensitivity is enhanced by 4 orders of magnitude as compared to measurements using thermally prepolarized samples at $1.6 \mathrm{~T}$. While inductive detection at zero field is generally insensitive, the optical magnetometer enables the acquisition of information-rich $J$-coupling spectra that can be used for chemical identification and fingerprinting, as demonstrated by our results and their agreement with numerical simulation and theory (see SI). The combination of nonperturbative hyperpolarization and detection by optical methods that we demonstrate here will broaden the applicability of low and zero-field NMR in chemical analysis. NH-PHIP works on a wide and still growing range of analytes. $^{4,37,38}$ The presented analysis suggests that all $\mathrm{NH}$ PHIP substrates are polarizable and detectable under identical zero-field conditions. In the future, we expect the combination of sensitive optical magnetometers with general hyperpolarization techniques such as NH-PHIP to enable chemical analysis in locations not accessible by traditional bulky and expensive NMR technology, thereby making NMR more widely accessible.

\section{Table 1. Signal-to-Noise Ratio (SNR) of the Largest Spectral Peak at $15.6 \mathrm{~Hz}$ as a Function of Analyte Concentration ${ }^{a}$}

$\begin{array}{cccccc}\text { concentration } & 6 \mathrm{mM} & 12 \mathrm{mM} & 40 \mathrm{mM} & 80 \mathrm{mM} & 160 \mathrm{mM} \\ \text { SNR } & 20 \pm 20 & 160 \pm 20 & 300 \pm 20 & 280 \pm 20 & 290 \pm 20\end{array}$

${ }^{a}$ Above $40 \mathrm{mM}$ pyridine concentration, we observe constant SNR of about 300. All identifying spectral features have SNR $>1$ for concentrations down to $6 \mathrm{mM}$. In these experiments, the noise floor was about $\sim 1 \mathrm{nG}$. 


\section{ASSOCIATED CONTENT}

\section{S Supporting Information}

Further experimental details; development of a two-spin model for NH-PHIP at zero field; comparison of the two-spin model, experiment and simulation; explanations of the simulations and for the choice of $J$-coupling values employed therein. This material is available free of charge via the Internet at http:// pubs.acs.org.

\section{AUTHOR INFORMATION}

\section{Corresponding Author}

pines@berkeley.edu, tho.theis@gmail.com

\section{Notes}

The authors declare no competing financial interest.

\section{ACKNOWLEDGMENTS}

Research was supported by the U.S. Department of Energy, Office of Basic Energy Sciences, Division of Materials Sciences and Engineering under Contract No. DE-AC02-05CH11231 (T. Theis, G. Kervern, J. W. Blanchard, P. Ganssle, M. C. Butler, A. Pines), and by the National Science Foundation under award \#CHE-0957655 (D. Budker and M. P. Ledbetter). We thank Vikram S. Bajaj for final edits to this manuscript and for many helpful discussions. We also thank Stephan Appelt and Ilya Kuprov for stimulating ideas and helpful conversations.

\section{REFERENCES}

(1) Weitekamp, D. P.; Bowers, R. C. J. Am. Chem. Soc. 1987, 109, 5541 .

(2) Natterer, J; Bargon, J. Prog. Nucl. Magn. Reson. Spectrosc. 1997, 31, 293.

(3) Duckett, S. B.; Wood, N. J. Coord. Chem. Rev. 2008, 252, 2278.

(4) Adams, R. W.; Aguilar, J. A.; Atkinson, K. D.; Cowley, M. J.; Elliott, P. I. P.; Duckett, S. B.; Green, G. G. R.; Khazal, I. G.; LopezSerrano, J.; Williamson, D. C. Science 2009, 323, 1708.

(5) Adams, R. W.; Duckett, S. B.; Green, R. a; Williamson, D. C.; Green, G. G. R. J. Chem. Phys. 2009, 131, 194505.

(6) Atkinson, K. D.; Cowley, M. J.; Elliott, P. I. P.; Duckett, S. B.; Green, G. G. R.; López-Serrano, J.; Whitwood, A. C. J. Am. Chem. Soc. 2009, 131, 13362 .

(7) Blümich, B.; Casanova, F.; Appelt, S. Chem. Phys. Lett. 2009, 477, 231.

(8) Abragam, A.; Goldman, M. Rep. Prog. Phys. 1978, 41, 395.

(9) Maly, T.; Debelouchina, G. T.; Bajaj, V. S.; Hu, K.-N.; Joo, C.-G.; Mak-Jurkauskas, M. L.; Sirigiri, J. R.; Wel, P. C. A.; van der; Herzfeld, J.; Temkin, R. J.; Griffin, R. G. J. Chem. Phys. 2008, 128, 052211.

(10) Goez, M. Annu. Rep. NMR Spectrosc. 2009, 66, 77.

(11) Walker, T. G.; Happer, W. Rev. Mod. Phys. 1997, 69, 629.

(12) Raftery, D. Annu. Rep. NMR Spectrosc. 2006, 57, 205.

(13) Navon, G.; Song, Y.-Q.; Room, T.; Appelt, S.; Taylor, R. E.; Pines, A. Science 1996, 271, 1848.

(14) Bowers, C. R. Sensitivity Enhancement Utilizing Parahydrogen. Encyclopedia of Nuclear Magnetic Resonance, Online; Grant, D. M.; Harris, R. K., Eds.; John Wiley \& Sons, Ltd.: Chichester, U.K., 2007; pp 1-19.

(15) Duckett, S. B.; Sleigh, C. J. Prog. Nucl. Magn. Reson. Spectrosc. 1999, 34, 71.

(16) Korchak, S. E.; Kiryutin, a. S.; Ivanov, K. L.; Yurkovskaya, a. V.; Grishin, Y. a.; Zimmermann, H.; Vieth, H.-M. Appl. Magn. Reson. 2010, 37,515 .

(17) Lingwood, M. D.; Ivanov, I. a; Cote, A. R; Han, S.-I. J. Magn. Reson. 2010, 204, 56.

(18) Halse, M. E.; Callaghan, P. T. J. Magn. Reson. 2008, 195, 162.

(19) Zotev, V. S.; Owens, T.; Matlashov, A. N.; Savukov, I. M.; Gomez, J. J.; Espy, M. a J. Magn. Reson. 2010, 207, 78.
(20) Ivanov, K. L.; Miesel, K.; Yurkovskaya, A. V.; Korchak, S. E.; Kiryutin, A. S.; Vieth, H.-M. H.-M. Appl. Magn. Reson. 2006, 30, 513.

(21) Vermeersch, G.; Marko, J.; Febvay-Garot, N.; Caplain, S.; Lablache-Combier, A. J. Chem. Soc., Perkin Trans. 2 1984, 2027, 2027.

(22) Sarvarov, F. S.; Salikhov, K. M.; R.Z., S. Chem. Phys. 1976, 16, 41.

(23) Appelt, S.; Haesing, F. .; Baer-Lang, S.; Shah, N. .; Blümich, B. Chem. Phys. Lett. 2001, 348, 263.

(24) Theis, T.; Ganssle, P.; Kervern, G.; Knappe, S.; Kitching, J.; Ledbetter, M. P.; Budker, D.; Pines, A. Nat. Phys. 2011, 7, 571.

(25) Gong, Q.; Gordji-nejad, A.; Blumich, B.; Appelt, S. Anal .Chem. 2010, 82, 7078 .

(26) Appelt, S.; Kühn, H.; Häsing, F. W.; Blümich, B. Nat. Phys. 2006, 2, 105

(27) Appelt, S.; Häsing, F. W.; Kühn, H.; Sieling, U.; Blümich, B. Chem. Phys. Lett. 2007, 440, 308.

(28) McDermott, R.; Trabesinger, A. H.; Muck, M.; Hahn, E. L.; Pines, A.; Clarke, J. Science 2002, 295, 2247.

(29) Greenberg, Y. Rev. Mod. Phys. 1998, 70, 175.

(30) Savukov, I. M.; Zotev, V. S.; Volegov, P. L.; Espy, M. A.; Matlashov, A. N.; Gomez, J. J.; Kraus, R. H. J. Magn. Reson. 2009, 199, 188

(31) Ledbetter, M. P.; Crawford, C. W.; Pines, A.; Wemmer, D. E.; Knappe, S.; Kitching, J.; Budker, D. J. Magn. Reson. 2009, 199, 25.

(32) Budker, D.; Romalis, M. Nat. Phys. 2007, 3, 227.

(33) Savukov, I.; Romalis, M. Phys. Rev. Lett. 2005, 94, 1.

(34) Ledbetter, M. P.; Savukov, I. M.; Budker, D.; Shah, V.; Knappe, S.; Kitching, J.; Michalak, D. J.; Xu, S.; Pines, A. Proc. Natl. Acad. Sci. U.S.A. 2008, 105, 2286.

(35) Ledbetter, M. P.; Theis, T.; Blanchard, J. W.; Ring, H.; Ganssle, P.; Appelt, S.; Blumich, B.; Pines, A.; Budker, D. Phys. Rev. Lett. 2011 $107,107601$.

(36) Glöggler, S.; Emondts, M.; Colell, J.; Müller, R.; Blümich, B.; Appelt, S. Analyst 2011, 136, 1566.

(37) Cowley, M. J.; Adams, R. W.; Atkinson, K. D.; Cockett, M. C. R.; Duckett, S. B.; Green, G. G. R.; Lohman, J. a B.; Kerssebaum, R.; Kilgour, D.; Mewis, R. E. J. Am. Chem. Soc. 2011, 133, 6134.

(38) Glöggler, S.; Müller, R.; Colell, J.; Emondts, M.; Dabrowski, M.; Blümich, B.; Appelt, S. Phys. Chem. Chem. Phys. 2011, 13, 13759.

(39) Lichter, R. L.; Roberts, J. D. J. Am. Chem. Soc. 1971, 93, 5218. (40) Jakobsen, H. J.; Yang, P.-I.; Brey, W. S. Org. Magn. Reson. 1981, 17,290 